\title{
SOME HOMOLOGICAL PROPERTIES OF SKEW $P B W$ EXTENSIONS ARISING IN NON-COMMUTATIVE ALGEBRAIC GEOMETRY
}

\author{
OSWALDo LEZAMA \\ Helbert Venegas \\ Seminario de Álgebra Constructiva - SAC ${ }^{2}$ \\ Departamento de Matemáticas \\ Universidad Nacional de Colombia, Sede Bogotá \\ e-mail: jolezamas@unal.edu.co
}

\begin{abstract}
In this short paper we study for the skew $P B W$ (Poincar-Birkhoff-Witt) extensions some homological properties arising in non-commutative algebraic geometry, namely, Auslander-Gorenstein regularity, Cohen-Macaulayness and strongly noetherianity. Skew $P B W$ extensions include a considerable number of non-commutative rings of polynomial type such that classical $P B W$ extensions, quantum polynomial rings, multiplicative analogue of the Weyl algebra, some Sklyanin algebras, operator algebras, diffusion algebras, quadratic algebras in 3 variables, among many others. Parametrization of the point modules of some examples is also presented.
\end{abstract}

Keywords: Auslander regularity condition, Cohen-Macaulay rings, strongly noetherian algebras, skew $P B W$ extensions, filtered-graded rings, point modules.

2010 Mathematics Subject Classification: Primary: 16S38; Secondary: 16W50, 16S80, $16 \mathrm{~S} 36$.

\section{REFERENCES}

[1] J.P. Acosta, C. Chaparro, O. Lezama, I. Ojeda and C. Venegas, Ore and Goldie theorems for skew PBW extensions, Asian-European J. Math. 6 (2013). doi:10.1142/S1793557113500617

[2] J.P. Acosta, O. Lezama and M.A. Reyes, Prime ideals of skew PBW extensions, Revista de la Unión Matemtica Argentina 56 (2015) 39-55.

[3] K. Ajitabh, S.P. Smith and J.J. Zhang, Injective resolutions of some regular rings, J. Pure Appl. Algebra 140 (1999) 1-21. doi:10.1016/S0022-4049(99)00049-3 
[4] V. Artamonov, Quantum Polynomials (WSPC Proceedings, 2008).

[5] V. Artamonov, Serre's quantum problem, Russian Math. Surveys 53 (1998) $657-730$. doi:10.1070/RM1998v053n04ABEH000056

[6] M. Artin, L.W. Small and J.J. Zhang, Generic flatness for strongly Noetherian algebras, J. Algebra 221 (1999) 579-610. doi:10.1006/jabr.1999.7997

[7] M. Artin, J. Tate and M. Van den Bergh, Some algebras associated to automorphisms of elliptic curves, The Grothendieck Festchrift, Vol. I, Birkhuser Boston (1990) 33-85. doi:10.1007/978-0-8176-4574-8-3

[8] M. Artin and J.J. Zhang, Noncommutative projective schemes, Adv. Mah. 109 (1994) 228-287. doi:10.1006/aima.1994.1087

[9] A. Bell and K. Goodearl, Uniform rank over differential operator rings and PoincarBirkhoff-Witt extensions, Pacific J. Math. 131 (1988) 13-37. doi:10.2140/pjm.1988.131.13

[10] J.E. Björk, Rings of Differential Operators, North-Holland Mathematical Library, vol. 21 (North-Holland Publishing Co., Amsterdam-New York, 1979).

[11] J.E. Björk, The Auslander Condition on Noetherian Rings, Sm. d'algbre P. Dubreil et M.-P. Malliavin, Lect. Notes Math. 1404 (1989) 137-173.

[12] J. Bueso, J. Gomez-Torrecillas and A. Verschoren, Algorithmic Methods in Noncommutative Algebra: Applications to Quantum Groups (Kluwer, 2003).

[13] J. Gomez-Torrecillas, Basic Module Theory over Non-Commutative Rings with Computational Aspects of Operator Algebras, in: M. Barkatou et al. (Eds.), AADIOS 2012, Lecture Notes in Computer Science 8372 (2014) 23-82.

[14] J. Gomez-Torrecillas and F.J. Lobillo, Global homological dimension of Multifiltered rings and quantized enveloping algebras, J. Algebra 225 (2000) 522-533. doi:10.1006/jabr.1999.8101

[15] J. Gomez-Torrecillas and F.J. Lobillo, Auslander-regular and Cohen-Macaulay Quantum groups, Algebras and Representation Theory 7 (2004) 35-42. doi:10.1023/B:ALGE.0000019384.36800.fa

[16] E.K. Ekström, The Auslander condition on graded and filltered noetherian rings, Sminaire Dubreil-Malliavin 1987-88, Lect. Notes Math. 1404 (Springer Verlag, 1989) $220-245$.

[17] G. Krause and T.H. Lenagan, Growth of algebras and Gelfand-Kirillov dimension, 2nd ed., Graduate Studies in Mathematics, 22 (AMS, Providence, USA, 2000).

[18] H. Kredel, Solvable Polynomial Rings (Shaker, 1993). 
[19] T. Levasseur, Some properties of non-commutative regular rings, Glasglow J. Math. 34 (1992) 277-300. doi: $10.1017 / \mathrm{S} 0017089500008843$

[20] T. Levasseur and J.T. Stafford, The quantum coordinate ring of the special linear group, J. Pure Appl. Algebra 86 (1993) 181-186. doi:10.1016/0022-4049(93)90102-Y

[21] O. Lezama and C. Gallego, Gröbner bases for ideals of sigma-PBW extensions, Communications in Algebra 39 (2011) 50-75. doi:10.1080/00927870903431209

[22] O. Lezama and M. Reyes, Some homological properties of skew PBW extensions, Communications in Algebra 42 (2014) 1200-1230. doi:10.1080/00927872.2012.735304

[23] O. Lezama and E. Latorre, Non-commutative algebraic geometry of semi-graded rings, arXiv:1605.09057 [math.RA].

[24] V. Levandovskyy, Non-Commutative Computer Algebra for Polynomial Algebras: Gröbner Bases, Applications and Implementation (Dissertation, University of Kaiserslautern, 2005).

[25] H. Li, Noncommutative Gröbner Bases and Filtered-Graded Transfer (Springer, 2002).

[26] H. Li, Non-Commutative Zariskian Rings, Ph.D Thesis (Universiteit Antwerpen, Antwerp, Belgium, 1990).

[27] H. Li and F.V. Oystaeyen, Zariskian filtrations, Comm. Algebra 17 (1989) 29452970 . doi:10.1080/00927878908823888

[28] A. Reyes, Gelfand-Kirillov dimension of skew PBW extensions, Revista Colombiana de Matemáticas 47 (2013) 95-111.

[29] A. Reyes, Ring and Module Theoretic Properties of $\sigma-P B W$ Extensions, Tesis de Doctorado (Universidad Nacional de Colombia, Bogotá, 2013).

[30] D. Rogalski, An introduction to non-commutative projective algebraic geometry, arXiv:1403.3065 [math.RA].

[31] A. Zaks, Injective dimension of semiprimary rings, J. Algebra 13 (1969) 73-89. doi:10.1016/0021-8693(69)90007-6

Received 19 July 2016

Revised 3 February 2017 
